\title{
Align Technology launches a new campaign to enhance orthodontists' brilliance
}

Align Technology, Inc. (NASDAQ: ALGN) has launched Your Brilliance Enhanced - a dedicated marketing campaign, celebrating the exemplary skills of orthodontic specialists.

Align's digital technology has been designed to amplify and enhance clinicians' specialism. The new campaign, Your Brilliance Enhanced, highlights the efficacy of combining the Invisalign system with the iTero scanner and end-to-end digitalisation, to enhance the brilliance of orthodontists and enable them to achieve more optimised outcomes. The campaign messaging celebrates the bilateral relationship, showcasing the analogy of a great musician and an instrument, which - in their hands - may be used to create a music masterpiece: 'Like the right instrument in the hands of a great musician, the Invisalign system and iTero scanners help enhance your orthodontic brilliance. Together, we can work to enhance your brilliance, so you can transform even more smiles and lives'.

Product innovation continues to drive Align's focus, even during these challenging times. Its aim remains the same: to help orthodontists improve the predictability of their clinical results to achieve brilliant outcomes, by harnessing the power of full practice digitalisation. Align has invested over $\$ 1$ billion in research and development over the last 23 years, enabling orthodontists to transform the smiles of adults, teens and children with the world's most advanced clear aligner system.

The Invisalign system, based on orthodontic principles, is clinically proven to achieve more predictable clinical results. The unparalleled expertise that comes from treating more than 9 million patients means greater accuracy, reliability and predictability. This, combined with end-to-end digitalisation, harnessing the iTero scanner digital workflow and tools, is helping many clinicians enhance their practice.

A new suite of digital innovations provides even greater flexibility, with advanced software to enable clinicians to move Invisalign digital treatment planning to the cloud. This makes ClinCheck 6.0 and

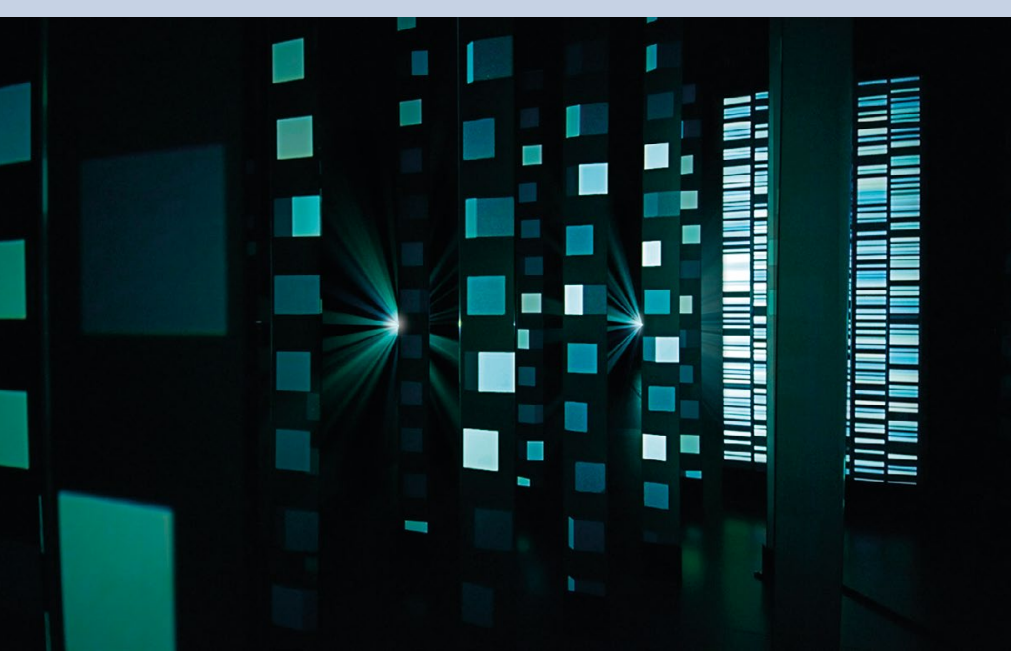

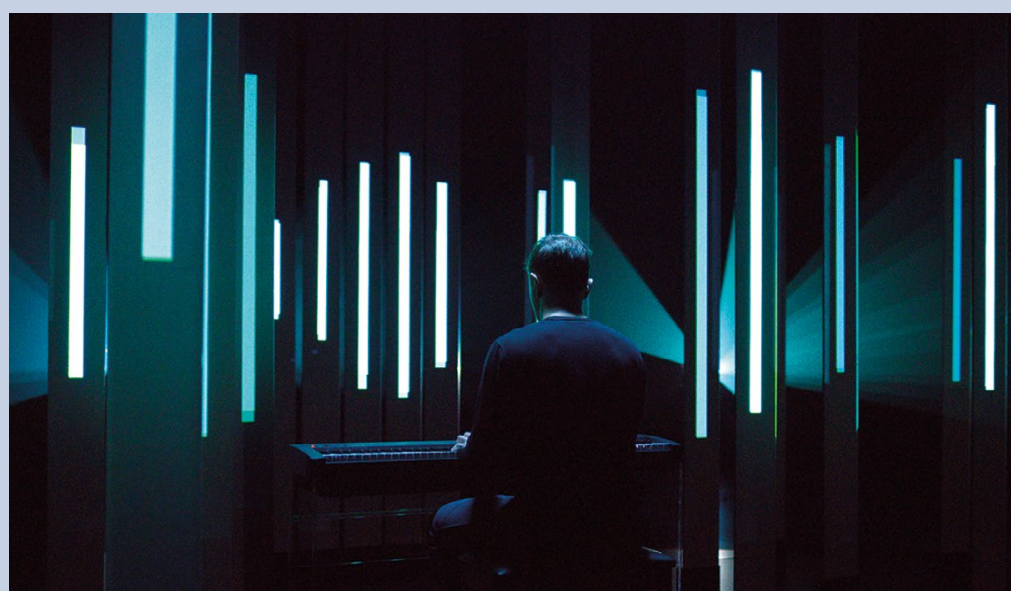

software features available to them anytime, anywhere, on a laptop, Mac, PC or iPad.

Orthodontists may also benefit from more powerful visualisation, thanks to the ClinCheck software's 'In-Face' Visualisation tool. This has been designed to help engage patients by showing them how their new smile could look in an image of their own face, as well as increasing their understanding of the Invisalign treatment. Clinicians can also plan treatments using a patient's own facial landmarks.

'This project is all about the brilliance of the orthodontist and how it's enhanced by what we bring to the table with Align Technology', says Lee Taylor, VP of Marketing, Align Technology EMEA. 'Imagine a world of seamless workflows and increased efficiency, with the control and accuracy you need for the outcomes you want time after time. We can support improved patient experience, driving practice growth. We're excited to help enhance clinical outcomes, practice digitalisation and professional development, through Align's campaign and ongoing learning and development initiatives.'

Making the time and space for professional development is just as important now as it ever has been. Align continues to invest in a wide range of educational activities through its Digital Academy, especially for orthodontists, to ensure the specialists continue to learn and develop in their profession.

'The message of the brilliance of the orthodontist being enhanced by digital technology has resonated well with the profession, and this combination of their brilliance - alongside our technical and digital knowledge - is transforming smiles and changing lives. At Align, we are committed to ongoing investment in innovation, with the intention of taking our products to the next level each year so that we can support orthodontists to deliver the best treatment outcomes. Our goal is to continue to facilitate the best clinical results and the best experience for patients - to enhance orthodontists' brilliance, added Abigail Lander, General Manager, Orthodontist Channel UKI.

Visit www.yourbrillianceenhanced.com for more information and to watch the campaign video. 